\title{
Simultaneous Radar, Aircraft and Meteorological Investigations of Clear Air Turbulence
}

\author{
Kenneth M. Glover, Roland J. Boucher, Hans Ottersten and Kenneth R. Hardy \\ Air Force Cambridge Research Laboralories, Bedford, Mass.
}

(Manuscript received 7 April 1969)

\begin{abstract}
The results of simultaneous studies of clear air turbulence (CAT) in the lower $15 \mathrm{~km}$ of the atmosphere by multi-wavelength radar, jet aircraft and special rawinsondes at the JAFNA radar facility at Wallops Island, Va., are reported. The most important finding is that for the particular aircraft and velocity used in these experiments, evcry clear air radar echo above $3 \mathrm{~km}$ is associated with aircraft reports of at least some perceptible degree of turbulence. Between the altitudes of 3 and $6 \mathrm{~km}$, all CAT is detected by the radars; however, the ability of the radars to detect weak CAT decreases with increasing altitude and only the more intense turbulence is detected above $12 \mathrm{~km}$. The indications are that strong CAT at high altitudes in the free atmosphere is generally associated with zones of increased refractive index variability and enhanced radar backscattering. Therefore, if radars of extreme sensitivity are employed, the useful range for CAT detection may be extended considerably and may possibly satisfy the requirements of an operational ground-based CAT detecting radar system. The vertical vector wind shear appears to be the most significant meteorological factor in specifying turbulent regions. A wind shear criterion $\geqslant 0.8 \times 10^{-2} \mathrm{sec}^{-1}$ applied to rawinsonde data specifies the presence or absence of turbulence correctly in $77 \%$ of all cases, including $100 \%$ of thc cases involving CAT greater than light.
\end{abstract}

\section{Introduction}

Since 1965, the sensitive multi-wavelength radar facility at Wallops Island, Va., has been used to investigate the nature of clear air radar echoes and in particular to evaluate the use of radar as a sensor for clear air turbulence (CAT). A number of the findings on the nature of clear air returns and CAT have already been reported (Hardy et al., 1966; Glover and Hardy, 1966; Atlas et al., 1966; Hicks et al., 1967; Hardy and Ottersten, 1968; Glover et al., 1968). This paper describes the results of simultaneous probes of the lower $15 \mathrm{~km}$ of the atmosphere for CAT by multi-wavelength radar, jet aircraft and special rawinsondes. The experiments were conducted during the period December 1967 through March 1968 during which a total of 58 flights of uninstrumented jet fighter-type aircraft (F-100, F-86 and F-4) were made in the region surrounding the Wallops radars. ${ }^{1}$

The use of radar for CAT detection has been prompted by the fact that the radar backscattering from clear air regions is related to the mean gradient of potential refractive index and to the degree of turbulence (Atlas et al., 1966; Ottersten, 1968). The sharper the original mean gradient and the more violent the turbulent mixing, the stronger the inhomogeneities

\footnotetext{
${ }^{1}$ During the period of the observations, the essential radar characteristics were: minimum detectable reflectivity at $15-\mathrm{km}$ range of $2.2 \times 10^{-15}, 1.3 \times 10^{-17}$ and $1.8 \times 10^{-18} \mathrm{~cm}^{-1}$; beamwidths of $0.21^{\circ}, 0.48^{\circ}$ and $2.9^{\circ}$; pulse lengths of $2.0,2.5$ and $5 \mu \mathrm{sec}$; and wavelengths of $3.2,10.7$ and $71.5 \mathrm{~cm}$, respectively.
}

that will be created; consequently, the radar scattering will be increased. There is, however, a large gap between the eddy sizes that affect aircraft and those that create the inhomogeneities detectable by radar. The radars are sensitive only to refractive index variations over scales of one-half the radar wavelength. Thus, for the $10.7-\mathrm{cm}$ and $71.5-\mathrm{cm}$ wavelength radars at Wallops Island, eddy sizes of about 5 and $35 \mathrm{~cm}$ are detected. On the other hand, aircraft are responsive mainly to turbulent eddies over scales ranging from about 10 to $1000 \mathrm{~m}$, the high values being applicable to heavy, high-speed aircraft. Because the energy at large eddy sizes is broken down into smaller and smaller eddies, radar measurements should provide some information on the turbulent kinetic energy at the larger scales.

For CAT generated by the local breakdown of wind shear in the free atmosphere, at least three conditions are necessary before turbulence of considerable intensity will develop (Hardy and Ottersten, 1968). First, the wind shear must progress beyond the point where the shearing stresses produce a fully developed turbulent flow despite the stabilizing influence of negative buoyancy. Second, some irregularity in the flow must be present in order to initiate the transformation of energy from the mean flow into turbulent kinetic energy. The third, and most basic, condition is that the initial lapse rate must be strongly stable in order to allow a strong vertical wind shear to build up before the turbulent motion is induced. The larger the original shear, the larger will be the turbulent kinetic energy that 
is extracted from the mean flow, and the more violent will be the turbulence and its effect on aircraft.

The preceding qualitative discussion may be described with the aid of the gradient Richardson number

$$
\mathrm{Ri}=\frac{g}{\theta}-\frac{\partial \theta / \partial z}{\beta^{2}}
$$

where $g$ is the acceleration due to gravity, $\partial \theta / \partial z$ the lapse rate of potential temperature, and $\beta$ the vertical wind shear. Here we are not concerned with negative Ri's because, for these cases, buoyancy forces are actually contributing to the turbulent kinetic energy. Such situations are not representative of the conditions which will be discussed. Except for turbulence associated with strong mountain lee waves, Richardson numbers are expected to determine the threshold of CAT, but the CAT intensity should depend upon the vertical vector wind shear. The Richardson number may be taken as an indicator of the relative strength of the stabilizing influence of negative buoyancy and the turbulence-inducing forces from wind shear. Large Ri's are indicative of stability, where every turbulent disturbance or irregularity will decay rapidly with time. Small Ri's indicate that a fully developed turbulent flow will be maintained; values $\lesssim 0.5$ are likely to be characteristic of developed turbulent flow (Penn and Pisinski, 1967). A low $\mathrm{Ri}$ is a necessary condition for strong turbulence; however, it is not a sufficient condition, because a low Ri may arise from a small $\partial \theta / \partial z$ in association with small $\beta$ values indicative of weak turbulence. Therefore, $\mathrm{Ri}$ cannot be used for any quantitative estimate of the degree of turbulence, but has to be regarded as a discriminator between regions where the turbulent flow is developed and regions of high stability where every turbulent disturbance is suppressed. The additional requirement for high turbulence intensities is large $\beta$ values, which we think cannot occur except in regions of high $\partial \theta / \partial z$.

The consequence of the above argument for radar measurements is that a correlation between the degree of turbulence and the magnitude of the mean vertical gradient of potential temperature should be apparent in the sense that large temperature gradients are necessary for the development of strong turbulence. Turbulence of considerable intensity should be associated with large stable mean vertical gradients of potential temperature and, consequently, with zones of enhanced small-scale temperature fluctuations created by turbulent mixing of the mean gradients. For the radar, this means that at high altitudes where the water vapor contribution to the refractive index variability is negligible, the backscattered signal intensity should be at least a crude quantitative measure of the degree of turbulence. Large temperature gradients may of course exist even though wind shears are small; in such a case, however, the turbulence is weak, and the

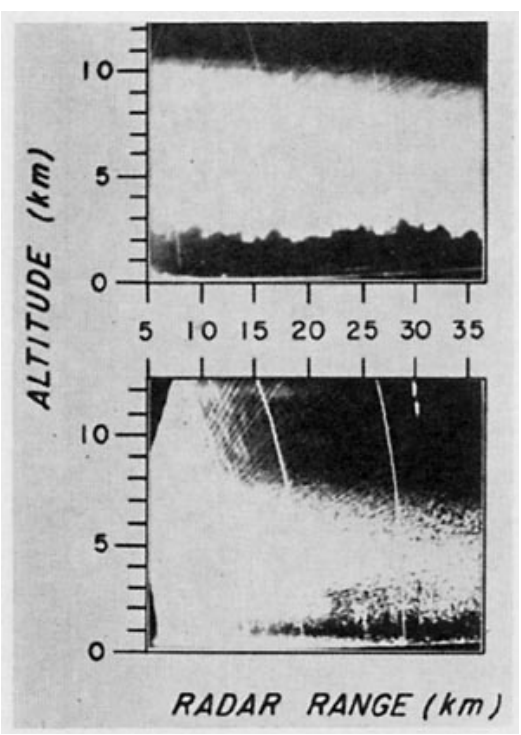

FIG. 1. Simultaneous range-height indicator photographs for the $3.2-\mathrm{cm}$ radar (top) and 71.5-cm radar (bottom) taken along an azimuth of $135^{\circ}$ for 0952 EST on 7 February 1968 at Wallops Island, Va. The echo between 2 and $10 \mathrm{~km}$ seen at $3.2-\mathrm{cm}$ wavelength and between 2 and $8 \mathrm{~km}$ at $71.5 \mathrm{~cm}$ wavelength is due to scattering from clouds or precipitation particles. The echo near 12 $\mathrm{km}$, seen only at the longer wavelength, is due to scattering from refractive index variations and coincides with aircraft reports of CAT.

small-scale inhomogeneities that give rise to radar signals are not intense enough to be detected.

\section{Measurement technique}

Each set of aircraft observations began with an ascent at a rate not exceeding $700 \mathrm{~m} \mathrm{~min}^{-1}$, from a height of 8 to $\sim 15 \mathrm{~km}$, along an $18-\mathrm{km}$ diameter spiral path. Occasionally, spirals from $3 \mathrm{~km}$ upward were obtained. About every 10-15 sec the pilots reported their altitude and qualitatively estimated the severity of any turbulence encountered; these data were recorded on tape throughout the flight. Upon completion of the spiral ascent, the aircraft were directed to the highest altitude either reported as or suspected of being turbulent, and a ground controller then vectored the aircraft along a horizontal racetrack pattern approximately $60 \mathrm{~km}$ from end to end. During horizontal flight, the aircraft generally maintained an indicated airspeed of $260 \pm 10$ $\mathrm{m} \mathrm{sec}-1$. Upon completing each racetrack, the aircraft decreased altitude by $300 \mathrm{~m}$ and then resumed the racetrack course until the entire turbulent altitude region was penetrated in closely spaced vertical intervals. Each flight consisted of these horizontal passes through as many such turbulent regions as possible during the approximately $1 \mathrm{hr}$ of flying time available. When necessary, aircraft soundings of the higher altitudes were given first priority.

The radar procedure consisted primarily in taking slow elevation scans $\left(1.0^{\circ} \mathrm{sec}^{-1}\right)$ at azimuths corresponding approximately to the azimuth of the aircraft as 


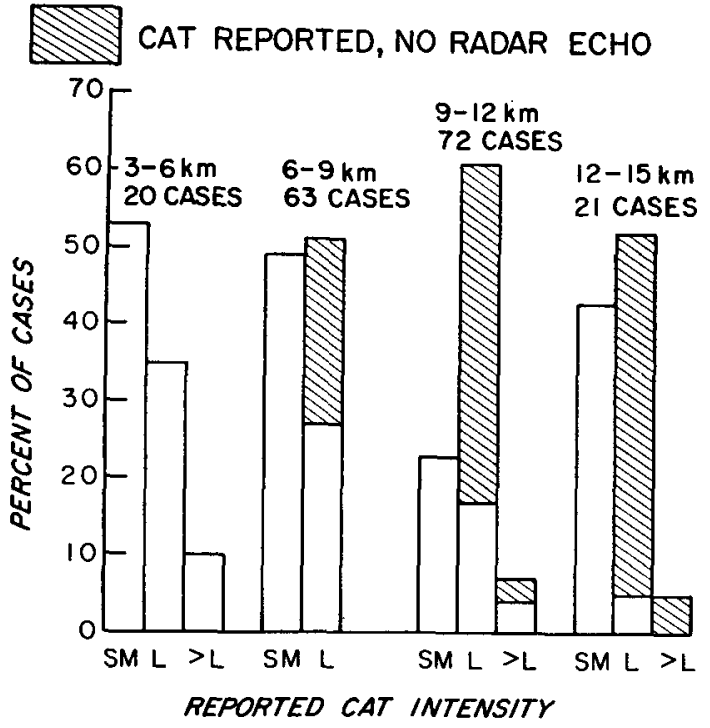

FIG. 2. Observed incidence of CAT and corresponding radar clear air echoes according to altitude and CAT intensity. Intensities are grouped into categories of smooth (SM), very very light to light (VVL-L), and greater than light $(>\mathrm{L})$. Every clear air radar echo above $3 \mathrm{~km}$ was associated with aircraft reports of perceptible turbulence.

reported by an ancillary tracking radar. From photographs of the range-height indicators (RHI) of the three radars, it was possible to determine both the location and extent of each clear air layer. An example of one such layer is illustrated in Fig. 1 which shows simultaneous photographs of the RHI displays for radar wavelengths of 3.2 and $71.5 \mathrm{~cm}$. The strong 3.2 $\mathrm{cm}$ echo between the heights of 2 and $10.5 \mathrm{~km}$ is due to the scattering from clouds or precipitation. With the $71.5-\mathrm{cm}$ radar, the corresponding echo is weaker because the backscattering from particles is reduced at the longer wavelength. The clear air radar layer is seen on the 71.5-cm photo at a height of $12 \mathrm{~km}$ out to a range of about $20 \mathrm{~km}$. At $3.2-\mathrm{cm}$ wavelength, this layer does not appear, and its absence at the shorter wavelength is sufficient evidence that the layer is caused by scattering from refractive index variations (Hardy et al., 1966). The aircraft confirmed that the sky was clear at a height of $12 \mathrm{~km}$.

Temperature, humidity and wind profiles representative of the conditions during the aircraft and radar observations were obtained from the ascent of a tandem pair of standard U. S. Weather Bureau radiosondes, with one sonde modified to provide a continuous record of temperature. With few exceptions, the rawinsonde observations were made during or within $1 \mathrm{hr}$ of the flight time.

\section{Results}

Thirty-nine of the 58 aircraft flights provided reports of CAT between the altitudes of 3 and $15 \mathrm{~km}$. In general, radar and meteorological observations were taken during each flight. There were, however, some flights for which one or more data sources were inoperative. As a result, there are some slight but insignificant differences in the flights compiled in the radar and meteorological results that follow.

\section{a. Radar and aircraft observations of CAT}

Reliable aircraft and radar data were obtained for 36 flights. Reports of CAT during these 36 flights were broken down into a relative scale based upon the pilot's comments of none, light, moderate and severe CAT, or combinations of these terms. Altitude profiles of turbulence intensity were constructed from these reports and compared with similar composite profiles of radar-echo height obtained from the radar photographs. The radar data were restricted to observations obtained when 1) the aircraft was within the maximum radar range of $37 \mathrm{~km}$, at which high-altitude clear air echoes are generally detected with the Wallops radars, and 2) there were no clouds within $300 \mathrm{~m}$ of the aircraft reporting position. From these data, 176 cases were obtained. Each case consisted of a $1-\mathrm{km}$ altitude interval characterized by either no CAT or the maximum CAT intensity in the interval, and the presence or absence of a corresponding clear air radar echo. For a confirmed radar detection, we required at least two successive photographs that showed radar echoes occurring at the same altitude as the aircraft report of CAT. No further consideration was given to a particular altitude interval unless all these criteria were satisfied.

The 176 cases of simultaneous radar and aircraft observations are summarized in Fig. 2, where the observed incidence of CAT and the presence or absence of corresponding clear air radar echoes are arranged according to altitude and CAT intensity intervals of smooth (SM), very very light to light (VVL-L), and greater than light $(>\mathrm{L})$. The unshaded regions represent those cases where both the aircraft and radars gave identical indications of either the existence or nonexistence of CAT, whereas the shaded areas represent those cases in which CAT was not detected by the radars. Probably the most obvious and the most important result in that for the particular aircraft and velocity used in these experiments, every clear air radar echo above $3 \mathrm{~km}$ has been associated with aircraft reports of at least some perceptible degree of turbulence. At lower altitudes, where the influence of water vapor upon the gradient of refractive index is strongest, this somewhat surprising result suggests that turbulence of a strength at least perceptible to an aircraft must be present to create detectable refractive index variations even if the mean gradient of refractive index is fairly strong.

Below $6 \mathrm{~km}$ all cases of CAT, regardless of intensity, were detected by the radars; however, the percentage of the cases detected decreased with increasing altitude 


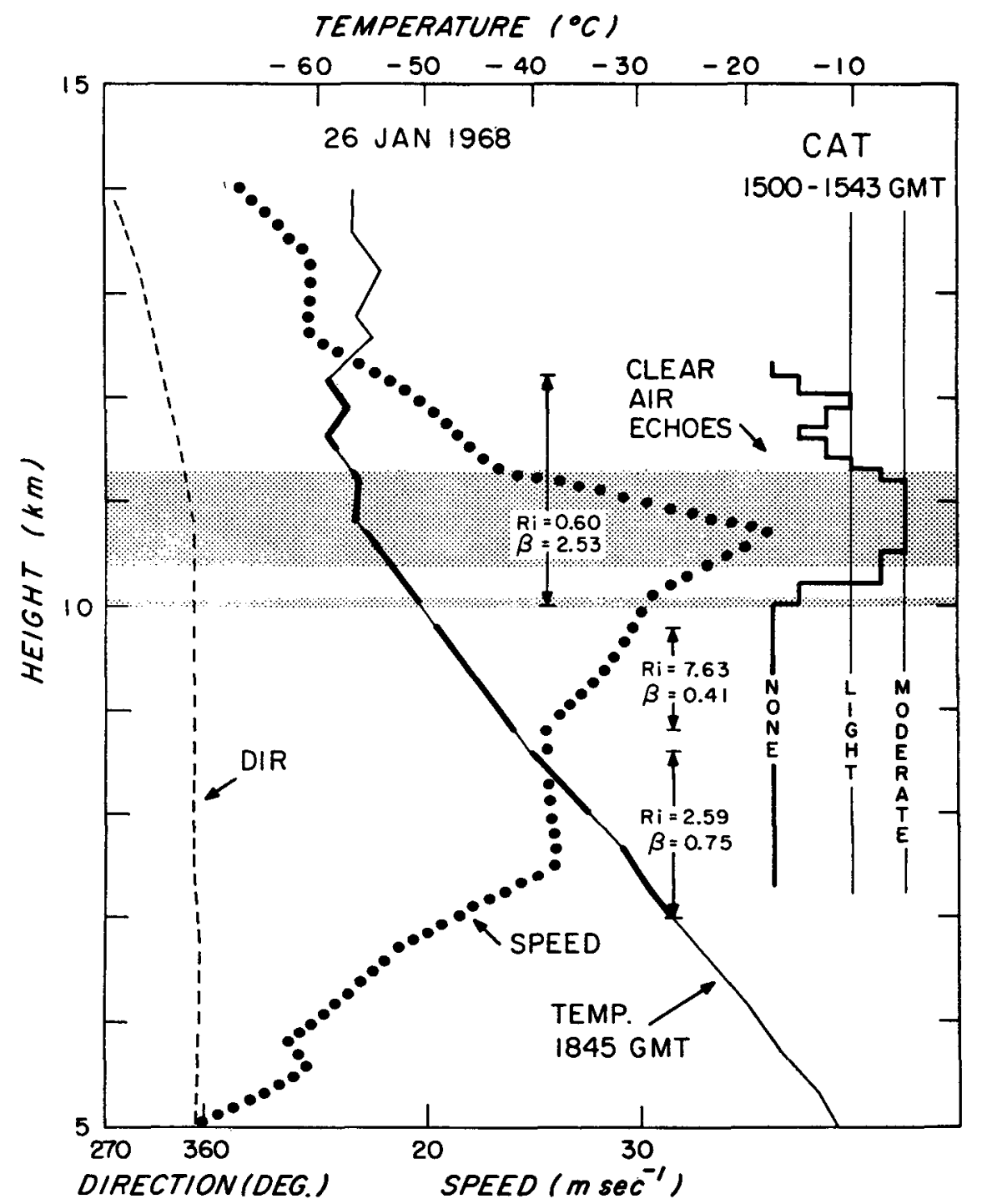

Fig. 3. Heights of radar echoes, reports of CAT, and meteorological soundings. Clear air radar echoes are indicated by the shaded layers, and the profile of aircraft encounters with CAT is given by the heavy line to the right of the sounding.

and only a small percentage of the cases of reported CAT were detected above $12 \mathrm{~km}$. This observed decrease in the radar detection capability is consistent with the expected influence of water vapor upon the mean gradient of refractive index.

The percentage of perceptible CAT cases detected by the radars within each of the individual altitude intervals was also computed by regrouping the turbulence intensities according to two categories: 1) less than light (VVL and VL), and ) 2 light or greater ( $\geq \mathrm{L}$ ). As shown in Table 1, these observations clearly demonstrate that the stronger CAT is consistently more likely to be detected than the weaker turbulence. It has been inferred on theoretical grounds that strong CAT at high altitudes will generally be associated with zones of enhanced small-scale variability in the atmospheric refractive index (Ottersten, 1968). Therefore, we should expect strong turbulence in the clear air to be more readily detectable than weak CAT. The experimental data are consistent with this thesis, and the implication

TABLE 1. Percentage of observed CAT detected by Wallops Island radars.

\begin{tabular}{lcccc}
\hline \hline & \multicolumn{4}{c}{ Altitude (km) } \\
$\begin{array}{c}\text { CAT } \\
\text { intensity }\end{array}$ & $3-6$ & $6-9$ & $9-12$ & $12-15$ \\
\hline $\begin{array}{c}\text { Less than } \\
\text { light } \\
\begin{array}{c}\text { Light or } \\
\text { greater }\end{array}\end{array}$ & 100 & 38 & 21 & 0 \\
\hline
\end{tabular}




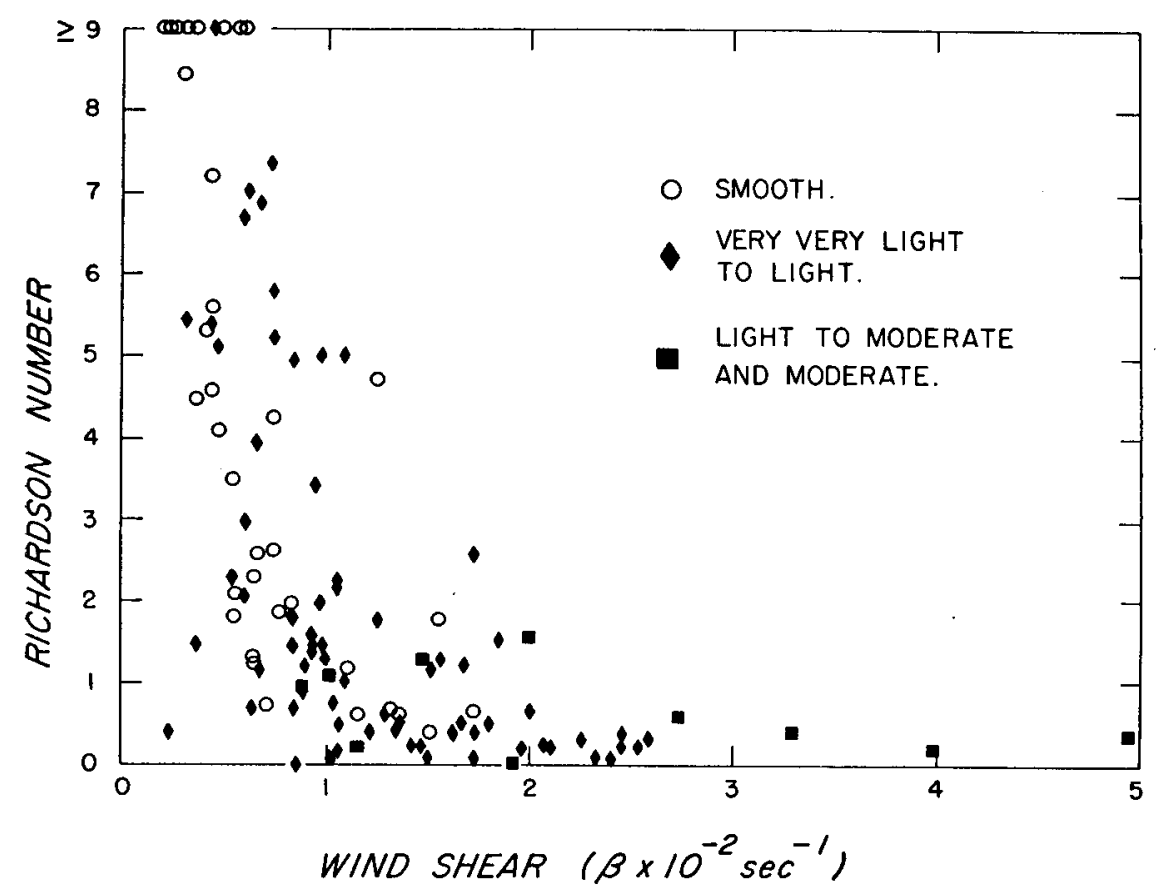

FIG. 4. Ri as a function of $\beta$ for each of the 117 cases. The key in the upper right identifies the cases according to the maximum CAT intensity reported by the aircraft.

is that more sensitive radars than those at Wallops Island should make it possible to detect most of the significant CAT up to altitudes of at least $15 \mathrm{~km}$.

Radar echoes from altitudes $\gtrsim 5 \mathrm{~km}$ were stratified, relatively thin $(0.1-1.5 \mathrm{~km})$, and-at $10.7-\mathrm{cm}$ wavelength-somewhat patchy (horizontal dimensions on the order of $5-30 \mathrm{~km}$ ). Moreover, the echoes were not particularly persistent, often lasting for less than a few tens of minutes. A similar horizontal stratification and patchiness was evident in the aircraft encounters with CAT. The transitory nature of both the radar and aircraft observations prohibited a detailed comparison of radar echo and CAT intensities.

\section{b. Meleorological analysis of $C A T$}

CAT reports from a total of 29 flights were correlated with the Richardson number $\mathrm{Ri}$ and the vertical vector wind shear $\beta$ derived from the special rawinsondes released from Wallops Island. For purposes of the meteorological analysis, the aircraft CAT reports were grouped into three categories: 1) smooth, 2) very very light to light, and 3) light to moderate and moderate. No CAT of intensity greater than moderate was encountered. From the aircraft and rawinsonde data, 127 cases were obtained, each case consisting of an altitude interval of about 1-2 $\mathrm{km}$, characterized by the presence or absence of aircraft-detected CAT. Most of the turbulence episodes were contained within single intervals. Each interval consisted of two or more subordinate layers a few hundred meters in depth, having quasihomogeneous lapse rates and wind shears. Values of
$\mathrm{Ri}$ and $\beta$ were computed for each layer on the basis of the original detailed rawinsonde records. The lowest Ri value and its corresponding $\beta$ within the interval were used to characterize the entire interval. With minor exceptions, the lowest $\mathrm{Ri}$ also corresponded to the highest $\beta$ in the interval.

An illustration of the method of analysis is given in Fig. 3 for 26 January 1968. The diagram shows the regions of CAT, the radar layers, and the rawinsonde data. The heavy segments of the temperature curve represent the layers used in the computation of $\mathrm{Ri}$, and the intervals or "cases" of CAT or no CAT with their corresponding $\mathrm{Ri}$ and $\beta$ are marked with vertical arrows. A profile of CAT intensity is shown at the right of the sounding. At a particular height, the indicated CAT intensity is the maximum intensity encountered by the aircraft, regardless of its horizontal position or the time spent at that altitude. For the case in Fig. 3 the tropopause is irregularly developed, but the main tropopause is just above $12 \mathrm{~km}$. The flight encountered two regions of CAT below this height. No definite radar detection was obtained from the region of light CAT near $12 \mathrm{~km}$, but the region of moderate CAT between 10.5 and $11.2 \mathrm{~km}$ was detected. In the region of light CAT, there is no obvious relationship between the CAT and the meteorological profiles; however, the lower region of moderate CAT is characterized by a stable lapse rate and strong shear.

As outlined in the introduction, Ri numbers are expected to determine the threshold of CAT, but CAT intensity should depend on the vertical vector-wind 
shear. Values of $\mathrm{Ri}$ and its dependent variable $\beta$ are compared in Fig. 4 for the 117 cases of turbulent or nonturbulent air. Aircraft reports are grouped according to the classes of turbulence intensity : smooth (36 cases); very very light through light ( 71 cases); and light to moderate and moderate (10 cases). In Fig. 4 , the occurrence of smooth air tends to be characterized by high $\mathrm{Ri}$ and turbulent air by low $\mathrm{Ri}$. There is, however, a region where turbulent and nonturbulent cases seriously overlap, thereby making the selection of a useful criterion somewhat difficult. In the analysis of the rawinsonde data, various criteria for the specification of regions of CAT were tested statistically. The criteria were based either on Ri numbers or on wind shears or on combinations of these values. The vertical vector wind shear alone appeared to be the most successful CAT indicator. Using a wind shear criterion of $\beta \geq 0.8$ $\times 10^{-2} \mathrm{sec}^{-1}$, it was found that an additional Richardson criterion would not improve the specification of turbulent regions.

The usefulness of the vertical vector-wind shear $\beta$ as an index of concurrent CAT is demonstrated in Fig. 5. In this figure are histograms displaying the incidence of CAT in 117 cases grouped into three categories of turbulence intensity and three categories of wind shear. From Fig. 5 it is evident that both the incidence and intensity of CAT increases sharply as the vertical wind shear increases. At $\beta \leq 0.8 \times 10^{-2} \mathrm{sec}^{-1}$, smooth air predominates (60\% of the cases) and no turbulence greater than light was observed. As the shear increases, the incidence and intensity of CAT rises until at $\beta \geq 1.8 \times 10^{-2} \mathrm{sec}^{-1}$, all cases are turbulent and $32 \%$ are greater than light. With the particular aircraft and speed used in our experiment, the wind shear criterion of $\beta \geq 0.8 \times 10^{-2} \mathrm{sec}^{-1}$ applied to rawinsonde data specified the occurrence or nonoccurrence of perceptible CAT correctly in $77 \%$ of the entire group of 117 cases from 29 separate flights, including $78 \%$ of the turbulent cases and $100 \%$ of the cases involving CAT greater than light.

These results show good agreement with earlier results, especially those of Penn and Pisinski (1967), which have demonstrated good correlation between low Richardson numbers and the incidence of turbulence, and have also certainly revealed the importance of the vertical wind shear as an energy source for turbulence. Simultaneously, we must emphasize that the limited resolution in our temperature and wind data prevents a quantitative comparison with the results obtained by Penn and Pisinski. Standard rawinsonde measurements tend to underestimate temperature gradients and, in particular, vertical wind shear; thus, the limited resolution in rawinsonde wind profiles can prevent accurate estimates of the vertical wind shear over CAT height intervals which of ten do not exceed $100 \mathrm{~m}$ in thickness. Since the deduced shear values can be severely underestimated, the estimates of Richardson numbers will

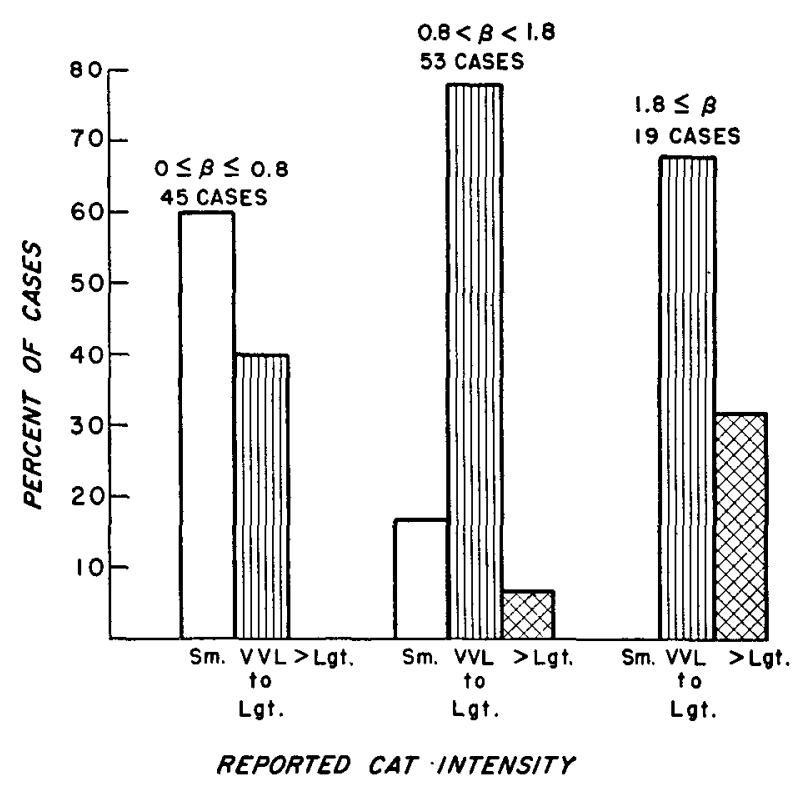

FIG. 5. Observed incidence of CAT according to vertical wind shear and CAT intensity. The magnitude of the shear is given in terms of $\beta \times 10^{-2} \mathrm{sec}^{-1}$.

be too high. Moreover, errors in the shear values are accentuated in the evaluation of the associated Richardson numbers; this may explain why, in our analysis, shear values alone are more reliable indicators of the CAT intensity.

Despite the limited resolution in rawinsonde data, it appears feasible to use wind shear criteria based on rawinsonde winds for the delineation of regions in the atmosphere, where the probability of aircraft encounters with significant turbulence is high. Further, it is interesting to note that all 14 cases of CAT with light-tomoderate or greater intensity occurred in altitude regions where the rawinsonde indicated a layer of increased stability. This is consistent with the thesis that strong vertical wind shear, which is a necessary condition for strong CAT, can develop only in the vicinity of layers of strongly stable temperature lapse rates. The consequence is that strong CAT will generally be associated with zones of increased variability in the refractive index. Since the radar backscattering from the clear air will be enhanced at these regions, it appears that radars of sufficient sensitivity should be a powerful tool in detecting CAT.

If radars of extreme sensitivity are employed, indications are that the useful range for CA'T detection may be extended considerably and may possibly satisfy the requirements of an operational ground-based CAT detecting radar system. It is still too early to advocate operational use of CAT-detecting radars but sensitive radars offer great potentials as research tools for the investigation of CAT. Further experiments should explore the spatial and temporal characteristics of high altitude clear air radar layers and their relationship to 
the distribution and life cycle of CAT. The results will determine if the remote detection of CAT with radar is feasible on an operational basis. A strong argument for radar is that the large-scale meteorological conditions conducive for CAT development favor the radar detection of CAT since, for these conditions, the turbulent mixing will enhance the small-scale refractive index fluctuations responsible for the radar scattering.

Acknowledgments. The research conducted at Wallops Island has been supported in part by the Laboratory Director's Fund, Air Force Cambridge Research Laboratories, and the National Aeronautics and Space Administration. The authors thank Mr. Jack Howard and the personnel of the JAFNA radar facility for their cooperation during all phases of this work; the personnel of the 113th and 175th Tactical Fighter Groups, Air National Guard, and A. F. Flight Dynamics MEDCAT Project for their skillful piloting of the aircraft ; and Mr. Edward F. Duquette of the authors' laboratory for his valuable assistance in the analysis of the observations. Finally, we greatly appreciate the contribution of Mr. Claude R. Landry who conducted most of the experiments and contributed significantly to the success of the program.

\section{REFERENCES}

Atlas, D., K. R. Hardy, K. M. Glover, I. Katz and T. G. Konrad, 1966: Tropopause detected by radar. Science, 153, 1110-1112. Glover, K. M., and K. R. Hardy, 1966: Dot angels: Insects and birds. Proc. 12th Radar Meteor. Conf., Boston, Amer. Meteor. Soc., 264-268.

—, R. J. Boucher, H. Ottersten and K. R. Hardy, 1968: Radar, aircraft and meteorological investigation of clear air turbulence. Proc. 13th Radar Meteor. Conf., Boston, Amer. Meteor. Soc., 242-247. (Also presented at the Natl. Conf. Atmospheric Turbulence, Amer. Meteor. Soc., 4-6 September, 1968, Boston, and at the USNC/URSI 1968 Fall Meeting, 10-12 September, 1968, Boston.)

Hardy, K. R., D. Atlas and K. M. Glover, 1966: Multiwavelength backscatter from the clear atmosphere. J. Geophys. Res., 71, $1537-1552$.

— and H. Ottersten, 1968: Radar and aircraft investigation of clear-air turbulence. Proc. 3rd Natl. Conf. Aerospace Meteor., New Orleans, 539-546.

Hicks, J. J., I. Katz, C. R. Landry and K. R. Hardy, 1967: Simultaneous radar and aircraft observations of clear-air turbulence. Science, 157, 808-809.

Ottersten, H., 1968: Theoretical aspects on C.AT detection by radar. Proc. 13th Radar Meteor. Conf., Boston, Amer. Meteor. Soc., 252-257.

Penn, S., and T. A. Pisinski, 1967: Mesoscale structure of the atmosphere in regions of clear air turbulence. Air Force Cambridge Res. Labs., Rept. AFCRL-67-0115, AFSG No 190, 85 pp. 